\title{
Electrical properties of Ge-Sb-Te-Bi Thin Films for Phase Change Memory Application
}

\author{
Alexey Sherchenkov ${ }^{1, a}$, Petr Lazarenko, b ${ }^{1,}$ Alexey Babich ${ }^{1, c}$, \\ Dmitriy Terekhov ${ }^{1, d}$ Sergey Kozyukhin ${ }^{2,}$ e \\ ${ }^{1}$ National Research University of Electronic Technology, Moscow, 124498, Russia \\ ${ }^{2}$ Kurnakov Institute of General and Inorganic Chemistry of the Russian Academy of Sciences, \\ Moscow, 119991, Russia \\ aaa_sherchenkov@rambler.ru, baka.jum@gmail.com, ${ }^{\mathrm{a}} \mathrm{drent} @ y a n d e x . r u$, \\ 'gsm.ads@yandex.ru, esergkoz@igic.ras.ru
}

Keywords: Phase Change Memory, Electrical Properties, Ge-Sb-Te

\begin{abstract}
In this study I-V characteristic, temperature dependence of resistivity, thermopower, were investigated for thin films on the basis of $\mathrm{Ge}_{2} \mathrm{Sb}_{2} \mathrm{Te}_{5}$ doped by Bi. Resistivities, ratio of the resistivities of amorphous and crystalline states, activation energies of conductivity, temperature of phase transition, Seebeck coefficient were estimated. Transport mechanisms in $\mathrm{Ge}_{2} \mathrm{Sb}_{2} \mathrm{Te}_{5}+\mathrm{Bi}$ thin films were analyzed.
\end{abstract}

\section{Introduction}

During the last years thin films of chalcogenide glassy semiconductors (CGS) of Ge-Sb-Te (GST) system was intensively studied due to their possible application in phase change memory (PCM) devices [1]. The work of such devices is based on rapid reversible phase transformations between amorphous and crystalline states, which take place in nanovolume of material under low-energy external influences. These phase transformations are accompanied by the abrupt changes in optical and electrical properties. The work of two types of memories is based on such abrupt changes of the materials - optical and electrical PCM.

At present the most widely investigated and used PCM materials have compositions along the $\mathrm{GeTe}-\mathrm{Sb}_{2} \mathrm{Te}_{3}$ pseudobinary line due to the rapid crystallization. Three intermetallic compounds exist on this pseudobinary line: $\mathrm{Ge}_{2} \mathrm{Sb}_{2} \mathrm{Te}_{5}, \mathrm{GeSb}_{2} \mathrm{Te}_{4}$ and $\mathrm{GeSb}_{4} \mathrm{Te}_{7}$ (so named GST materials). The most interesting of them is $\mathrm{Ge}_{2} \mathrm{Sb}_{2} \mathrm{Te}_{5}$, which exhibits appreciable stability at room temperature, high crystallization rate and good reversibility between amorphous and crystalline phases [1-4].

Nowadays phase change optical recording discs with different formats [CD-RW, DVD-RW, DVDRAM, and Blu-ray] have obvious commercial success. In addition, phase change electrical memory devices [phase-change random access memory (PRAM)] are considered as most promising candidates for the next generation of memories [1] due to non-volatility, low power consumption, fast operation speed, high endurance, extended scalability, low manufacturing cost.

However, despite of commercial success of optical PCM (CD, DVD, Blu-ray discs) electrical PCM technology must be improved, and effective methods for controlling electrical properties of PCM materials are needed. But controlling the electrical properties of CGS is a very complex problem because of the insensitivity of most chalcogenide glassy semiconductors to doping due to the Fermi level pinning. One of the ways to control chalcogenide properties is to dope these materials with isomorphic elements. For GST225 such an element is Bi. However, the effect of influence of bismuth doping on the electrical properties of this PCM material is not well understood.

Thus, the aim of this work was investigation of the influence of Bi doping phase transformation on the electro-physical properties, current-voltage characteristics, and transport mechanisms in $\mathrm{Ge}_{2} \mathrm{Sb}_{2} \mathrm{Te}_{5}$ thin films. 


\section{Experiment}

The initial $\mathrm{Ge}_{2} \mathrm{Sb}_{2} \mathrm{Te}_{5}$ alloys doped with different amounts of $\mathrm{Bi}(0.5,1$ and 3 wt. \%) were prepared with using of synthesis method described in [5]. Thin films were prepared by thermal evaporation in vacuum of the synthesized GST225. Substrate temperature during the deposition did not exceed $50{ }^{\circ} \mathrm{C}$.

X-ray diffraction (Rigaku D/MAX, $\mathrm{Cu} \mathrm{Ka} \mathrm{k}=0.15481 \mathrm{~nm}$ ) showed that synthesized materials has trigonal modification of $\mathrm{Ge}_{2} \mathrm{Sb}_{2} \mathrm{Te}_{5}$. Introduction of $\mathrm{Bi}$ leads to the appearance of trigonal modification of $\mathrm{Bi}_{2} \mathrm{Ge}_{2} \mathrm{Te}_{5}$, which indicates on the replacement of $\mathrm{Sb}$ by Bi. As-deposited thin films were amorphous.

Compositions of thin films were checked by Rutherford backscattering $\left(\mathrm{E}_{\mathrm{d}}=1.0, \mathrm{E}_{\alpha}=2.7 \mathrm{MeV}\right.$, $135^{\circ}$ scattering angle). It was shown that compositions of thin films were close to those of the synthesized materials.

For the measurements of the electro-physical properties and current-voltage characteristics planar structures containing $\mathrm{Al}$ electrodes with fixed interelectrode distances, and deposited upon them investigated thin films were fabricated on oxidized c-Si substrates.

Investigation of thermopower and determination of the type of conductivity were carried out on the samples containing Al electrodes fabricated on pyroceramics substrates and deposited upon electrodes investigated thin films. The distance between electrodes was $5 \mathrm{~mm}$, and temperature gradient was $5^{\circ} \mathrm{C}$.

The spectroscopic ellipsometry measurements were carried out using a variable angle ellipsometer (ELLIPSE-1881A) in the wavelength range of $380-1050 \mathrm{~nm}$ with a step of $10 \mathrm{~nm}$ at an incident angle of $70^{\circ}$.

\section{Results and Discussion}

The temperature dependencies of resistivity for GST225 + Bi thin films are shown in Fig. 1 and Table 1. The resistivity measurements showed that crystallization is accompanied by the drastic decrease of resistivity (see Fig. 1). Determined onset crystallization temperatures $\left(T_{\mathrm{x}}\right)$ for GST225 with different $\mathrm{Bi}$ content $\left(\mathrm{C}_{\mathrm{Bi}}\right)$ correlates with the data of XRD, optical measurements by spectrophotometry, and differential scanning calorimetry.

Crystallization is accompanied by the sharp drop of the resistivities. Ratio of the resistivities of amorphous and crystalline states exceeds $10^{3}$ (see Table 1), which is important for the reliable work of PCM.

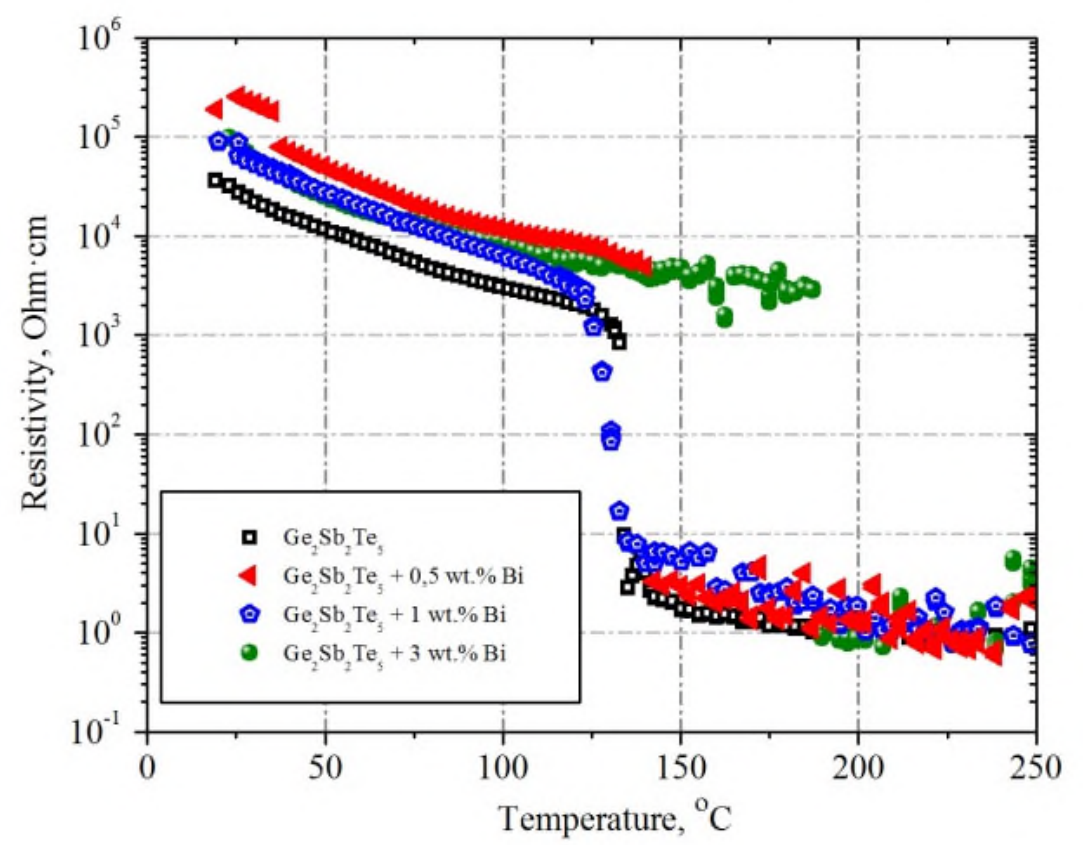

Fig. 1 Temperature dependences of resistivity for GST225 + Bi thin films. 
Measurements of current-voltage characteristics of the amorphous samples at different temperatures (from 293 to $373 \mathrm{~K}$ ) showed the existence of tree voltage ranges with different I-V dependencies indicating different transport mechanisms. Fig. 2 illustrates typical current-voltage characteristics at different temperatures.

For low electric field strength $\left(\mathrm{E}<10^{3} \mathrm{~V} / \mathrm{cm}\right)$ region with Ohmic behavior was observed. Exponential temperature dependences of conductivity were established for all amorphous thin films at low voltages in the temperature range from 220 to $340 \mathrm{~K}$ (Fig. 3).

Table 1. Results of temperature dependence of resistivity measurements for the $\mathrm{Ge}_{2} \mathrm{Sb}_{2} \mathrm{Te}_{5}+\mathrm{Bi}$ thin films.

\begin{tabular}{c|c|c|c|c}
\hline $\mathrm{C}_{\mathrm{Bi}}$, wt. $\%$ & $\mathrm{~T}_{\mathrm{x}},{ }^{\circ} \mathrm{C}$ & $\rho_{25}, \Omega^{*} \mathrm{~cm}$ & $\rho_{200}, \Omega^{*} \mathrm{~cm}$ & $\rho_{25} / \rho_{200}$ \\
\hline 0 & 131.6 & $2.8 \cdot 10^{4}$ & 1.0 & $2.8 \cdot 10^{4}$ \\
\hline 0.5 & 140.3 & $2.5 \cdot 10^{5}$ & 0.8 & $1.4 \cdot 10^{5}$ \\
\hline 1 & 123.1 & $8.9 \cdot 10^{4}$ & 1.0 & $8.9 \cdot 10^{4}$ \\
\hline 3 & 187.7 & $8.3 \cdot 10^{4}$ & 0.9 & $9.1 \cdot 10^{4}$ \\
\hline
\end{tabular}

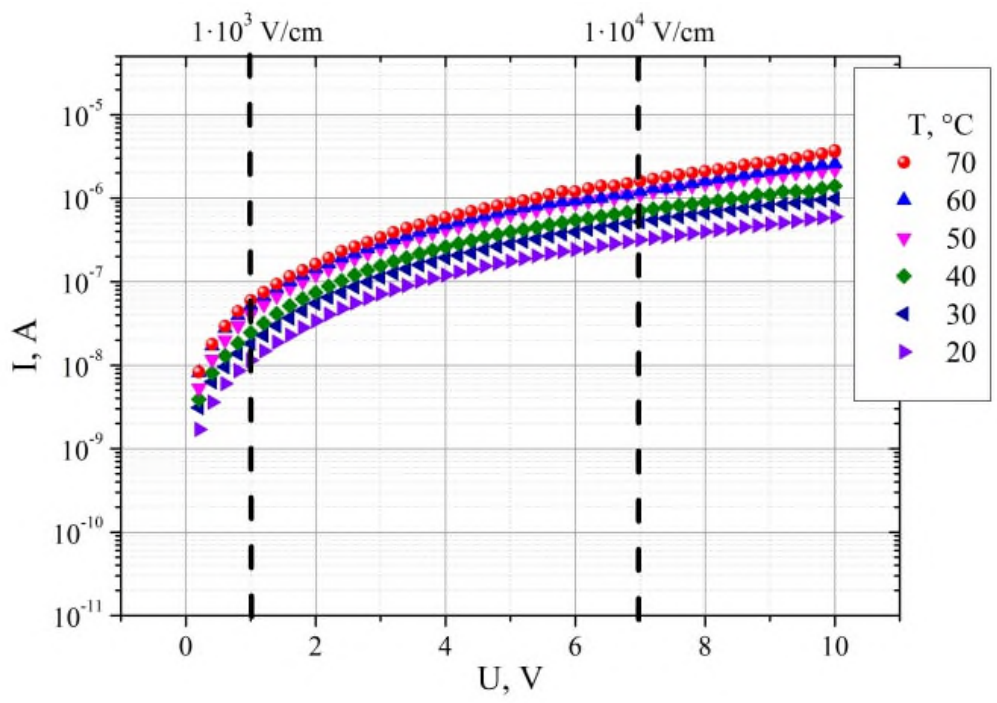

Fig. 2 CVC for the GST225 thin film.

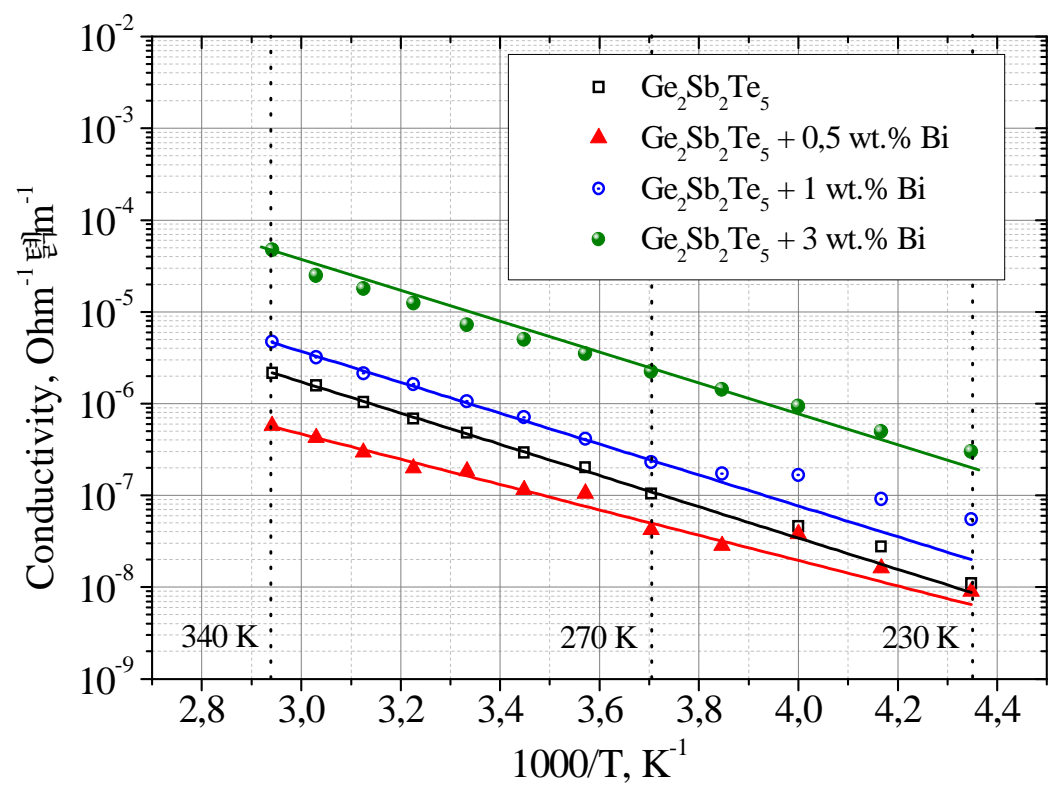

Fig. 3 Temperature dependencies of conductivity for amorphous thin films. 
Incorporation of $\mathrm{Bi}$ leads to the increase of the resistivity of amorphous films and the ratio of the resistivities of films in amorphous and crystalline states, which is important for the reliable work of PCM cells. The symbols $\rho_{25}$ and $\rho_{200}$ in the Table 1 represent resistivities of the amorphous films at $25^{\circ} \mathrm{C}$ and crystalline films at $200{ }^{\circ} \mathrm{C}$, respectively.

Activation energies of conductivity $\left(\mathrm{E}_{\mathrm{a}}\right)$ were calculated for all films (Table 2). Energy band gap $\left(\mathrm{E}_{\mathrm{g}}\right)$ and Urbach energy $\left(\mathrm{E}_{0}\right)$ for investigated compounds with different $\mathrm{Bi}$ content $\left(\mathrm{C}_{\mathrm{Bi}}\right)$ were obtained by spectrophotometry, and are presented in Table 2 .

The results showed that GST225 with 0.5 wt. \% of Bi has the maximum $\mathrm{E}_{\mathrm{g}}(0.65 \mathrm{eV})$ and minimum Urbach tails $(0.08 \mathrm{eV})$ indicating on the least disordered structure. Where in the $\mathrm{E}_{\mathrm{a}}$ in this case was slightly lower for GST225+ 0.5 wt. \% of Bi. Changes in the distribution of density of states in the mobility gap can be one of the possible explanations for this deviation. Maximum value of Urbach energy was observed for composition with 3 wt. $\% \mathrm{Bi}\left(\mathrm{E}_{0}=0.15 \mathrm{eV}\right)$. It can be due to the high mechanical stresses in the material matrix or phase separation at high concentration of bismuth. The energy diagram for GST225 thin film, and it's modification with Bi doping (Fig. 4) were designed with using of obtained results.

Results of thermopower measurements showed that holes are majority carriers in the investigated thin films. Seebeck coefficient for amorphous GST225 thin films is equal to $591 \mathrm{mcV} / \mathrm{K}$ and decreases with the temperature. Crystallization of the film is accompanied by the further sharp drop of Seebeck coefficient up to $115 \mathrm{mcV} / \mathrm{K}$. Addition of Bi leads to the increase of Seebeck coefficient ( $826 \mathrm{mcV} / \mathrm{K}$ for GST225+ $0.5 \mathrm{wt}$. \% of Bi amorphous thin film).

Table 2. Energy parameters for the amorphous $\mathrm{Ge}_{2} \mathrm{Sb}_{2} \mathrm{Te}_{5}+\mathrm{Bi}$ thin films.

\begin{tabular}{c|c|c|c|c|c}
\hline $\mathrm{C}_{\mathrm{Bi}}$, wt. \% & $\mathrm{E}_{\mathrm{a}}, \mathrm{eV}$ & $\mathrm{E}_{\mathrm{g}}, \mathrm{eV}$ & $\mathrm{E}_{0}, \mathrm{eV}$ & $\mathrm{E}_{\mathrm{t}}, \mathrm{eV}$ & $\mathrm{N}_{\mathrm{t}}, \mathrm{cm}^{-3}$ \\
\hline 0 & 0.29 & 0.61 & 0.13 & 0.28 & $8 \cdot 10^{14}$ \\
\hline 0.5 & 0.26 & 0.65 & 0.08 & 0.23 & $7 \cdot 10^{17}$ \\
\hline 1 & 0.30 & 0.53 & 0.09 & 0.30 & $3 \cdot 10^{15}$ \\
\hline 3 & 0.28 & 0.51 & 0.15 & 0.31 & $3 \cdot 10^{14}$ \\
\hline
\end{tabular}

Dual-channel model was proposed for chalcogenide glassious semiconductors [6]. According to this model p-type conductivity is formed by the charge carrier transport by delocalized states of the valence band, and localized states of the valence band tail. As the values of $E_{a}$ of the investigated thin films are close to the half of width of the mobility gap, the main contribution in the conductivity is introduced by the transport of charge carriers by the delocalized states.

For all compositions investigated in the middle range of $\mathrm{I}-\mathrm{V}$ characteristics power dependences of current on the applied voltage were established. Such dependences correspond to the space charge limited current (SCLC) and are also observed in disordered semiconductors when charge carriers are transferred by band states, and when they are captured on the discrete levels of localized states. With using the theories of Rose and Lampert [7] we estimated position of the trap level controlling transport mechanism $\left(\mathrm{E}_{\mathrm{t}}\right)$ and the density of states at the $\mathrm{E}_{t}$ level $\left(\mathrm{N}_{\mathrm{t}}\right)$. Results of estimations are presented in Table 2.

Estimated energy level $E_{t}$ for middle range is close to the value of the activation energy of conductivity for linear region. The maximum difference between $E_{a}$ and $E_{t}$ is observed for GST225 +0.5 wt. $\%$ of $\mathrm{Bi}(0.03 \mathrm{eV})$, which indicate on the largest shift of the position of level $\mathrm{E}_{\mathrm{t}}$ from the middle of the mobility gap to the top of the valence band. It may be connected with the maximum density of states for level $\mathrm{E}_{\mathrm{t}}\left(\mathrm{N}_{\mathrm{t}}\right)$, which was observed for GST225 with 0.5 wt. \% of Bi.

When applied voltage is low the holes injected from aluminum contact are captured by deep traps, but current-voltage characteristic is linear until the average concentration of the injected charge carriers becomes comparable with the concentration of thermally generated carriers. With increasing of concentration of the injected holes in valence band the position of quasi-Fermi level begins to shift closer to the valence band. So, the position of the level determining activation energy for nonlinear range $\left(E_{t}\right)$ is somewhat closer to the top of the valence band than that for the linear range $\left(\mathrm{E}_{\mathrm{a}}\right)$. 


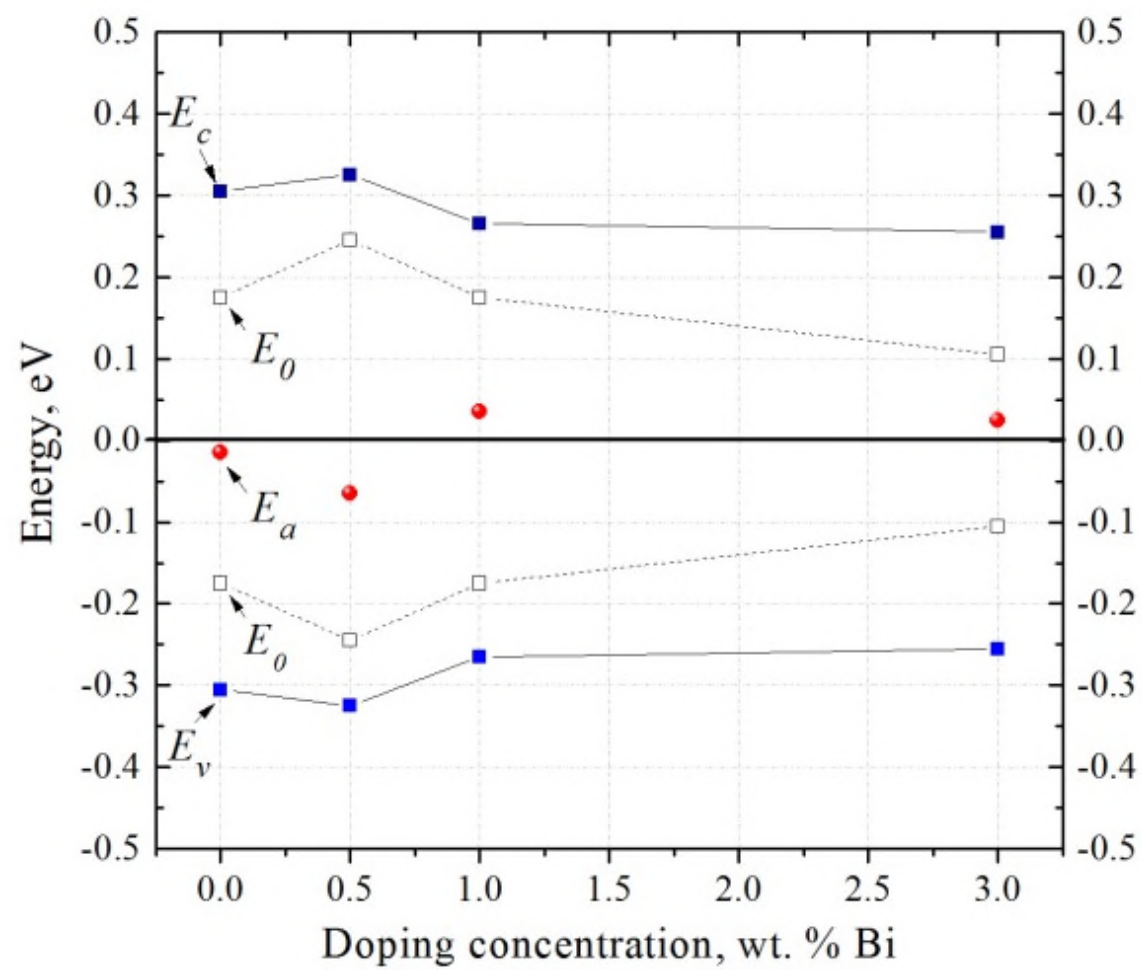

Fig. 4 Energy diagram for Bi doped GST225 thin films.

Thus, investigation of the influence of bismuth concentration on the parameters of GST225 thin films showed that the concentration dependences are not monotonic. The most significant deviations of parameters are observed for GST225 with 0.5 wt. $\%$ of Bi. This is reflected in the appearance of extremes on the concentration dependences. This effect can be explained by using percolation theory when at critical concentration formation of infinite cluster is accompanied by critical phenomena [8].

\section{Conclusion}

Thus, in this study I-V characteristics, temperature dependence of resistivity, thermopower were investigated for thin films on the basis of $\mathrm{Ge}_{2} \mathrm{Sb}_{2} \mathrm{Te}_{5}$ doped by $\mathrm{Bi}$. Resistivities, ratio of the resistivities of amorphous and crystalline states, activation energies of conductivity, temperature of phase transition, Seebeck coefficient were estimated. Introduction of bismuth to $\mathrm{Ge}_{2} \mathrm{Sb}_{2} \mathrm{Te}_{5}$ allows significantly expand the range of properties of thin films for phase change memory application. Investigation of the influence of bismuth concentration on the parameters of GST225 thin films showed that the concentration dependences are not monotonic. Introduction of the low concentration of bismuth ( $0.5 \mathrm{wt} . \%$ of $\mathrm{Bi})$ in GST225 thin films is accompanied by the deviation from monotonous concentration dependences of crystallization temperature, resistivity, width of mobility gap, Urbach energy, energy activation of conductivity, position of the traps, and distribution of density of states in the mobility gap.

\section{Acknowledgement}

This study was supported by Ministry of Education and Science of RF (project ID: RFMEFI57514X0096).

\section{References}

[1] W. B. Geoffrey et al. Phase change memory technology. J. Vacuum Sci. Technol. B. 28(2) (2010) 223- 262. 


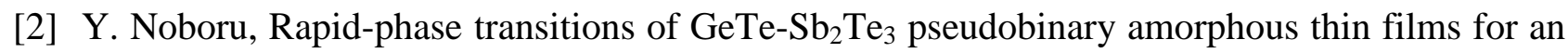
optical disk memory. J. Appl. Phys. 69 (1991) 2849-2856.

[3] H. S. P. Wong, S. Raoux, S. B. Kim, J. Liang, J. P. Reifenberg, B. Rajendran, M. Asheghi, K. E. Goodson, Phase change memory, Proc. IEEE. 98 (2012) 2201-2227.

[4] S. Raoux, W. Wełnic, D. Ielmini. Phase change materials and their application to nonvolatile memories. Chem. Rev. 110 (2010) 240-267.

[5] S. Kozyukhin, A. Sherchenkov, A. Babich, P. Lazarenko, H. P. Nguyen, O. Prikhodko. Peculiarities of Bi doping of Ge-Sb-Te thin films for PCM devices. Canadian J. Phys. 92(7/8) (2014) 684-689.

[6] P. Nagels. Electronic transport in amorphous semiconductors. Topic Appl. Phys. 36 (1985) 113-158.

[7] M. Lampert. Current Injection in Solids. NY and London: Academic Press Inc, 1970.

[8] E. Rogacheva. Self-organization processes in impurity subsystem of solid solutions. J. Phys. Chem. Solids. 64 (2003) 1579-1583. 